\title{
Wünsche und Bedürfnisse Schwangerer und Gebärender an den Geburtsort
}

Karolina Luegmair, Hebamme, B.Sc. Psychologie, M.Sc. Midwifery, Fahrenzhausen

\begin{abstract}
Zahlreiche Studien gehen in jüngster Zeit der Frage nach, wie Frauen ihre Geburt erleben und welche Wünsche sie an den Geburtsort mitbringen. Zur Entwicklung eines Auswertungsverfahrens für die österreichischen Daten des Babies Born Better Survey [1] hat die Autorin einen Literaturüberblick über bereits erfolgte Untersuchungen zur Zufriedenheit der Frauen mit erlebten Betreuungs-Bedingungen während der Geburt erstellt [2].
\end{abstract}

\begin{abstract}
Der Fokus der Untersuchungen des Geburtserlebnisses von Frauen richtete sich in der Vergangenheit immer mehr auf individuelles Erleben im Rahmen der Gegebenheiten. Um individuelles Erleben abzubilden, eignen sich besonders gut qualitative Erhebungsmethoden. Doch - wie in vielen Bereichen der Medizin - beschränken sich auch in der geburtshilflichen Forschung die Designs häufig auf die quantitativen Wege bzw. es ist ein deutlicher Mangel an Literatur festzustellen, die den Frauen eine eigene Stimme gibt, um ihre Erlebnisse zu berichten [3]. Daher ist ein großer Bedarf an Forschung zu vermerken, welche die individuelle Perspektive der Frau untersucht. Zwar ist vor allem durch die Hebammenforschung der Weg der qualitativen Forschung beliebter geworden und die Häufigkeit dieser Untersuchungen gestiegen, geschuldet dem aufwändigen Auswertungsverfahren beschränkt sich die Zahl der befragten Frauen aber häufig auf kleine Stichproben.
\end{abstract}

\section{Untersuchung des Geburtsortes - nötige Perspektiven}

Beim tatsächlichen Erleben der Rahmenbedingungen einer Geburt schließlich geht es selten ausschließlich um die Hebamme oder die (Klinik-)Umgebung - vielmehr sehen sich Frauen und ihre Begleitpersonen meist einer Vielzahl an Bedingungen gegenübergestellt, mit denen sie sich während der Geburt arrangieren müssen: Die Umgebung, das gesamte anwesende medizinische Personal, aber auch das eigene Erleben und nicht zuletzt die Gefühle von Freude und oft genug auch Sorge, eventuell sogar Angst. All diese Bedingungen (und sicher noch mehr) spielen in unterschiedlichen Ausprägungen in die individuelle Geburtserfahrung hinein. Und all diese Bedingungen werden in variierenden Anteilen dazu beitragen, ob die Geburt zu einem positiven Erlebnis wird.

Was also brauchen Frauen, um sich im Rahmen ihrer Geburt sicher zu fühlen? Welche Anforderungen haben sie an das Setting, das Personal, die gesamte Umgebung und womöglich auch an sich selbst? Dieser Frage wurde in den vergangenen Jahren vermehrt nachgegangen. Dabei wurde der Versuch, sie zu beantworten, häufig mit quantitativen Methoden (z.B. im Rahmen einer Fragebogenstudie), aber auch mit qualitativen Methoden (Interviews von Einzelpersonen) oder MixedMethods-Forschungsansätzen (Kombination qualitativer und quantitativer Verfahren) verfolgt.

Einige Beobachtungen und Erkenntnisse wiederholten sich: So waren die Themen der wahrgenommenen Kontrollierbarkeit der Situation, auch im Sinne von Informationen und Aufklärung, sowie Sicherheit und Vertrauen in Interventionen, die Unterstützung durch geburtshilfliches Personal und die Atmosphäre während der Geburt wichtig. Diese Faktoren ergaben sich als Extrakt aus einem Literaturüberblick über europäische und deutschsprachige Veröffentlichungen der vergangenen 10-15 Jahre, welcher die Wünsche und Bedürfnisse von Frauen im Rahmen ihrer Geburt betrachtet. Diese werden im Folgenden vorgestellt, sortiert in die drei gefundenen übergeordneten Dimensionen der Rahmenbedingungen, emotionalen Erfahrungen und erlebten peripartalen Betreuung.

\section{Rahmenbedingungen}

Zuerst sollen die äußeren Bedingungen des Geburtsortes betrachtet werden. Zu diesen zählen neben Faktoren der Erreichbarkeit und Infrastruktur - Tuschy et al. erwähnen beispielsweise die erwünschte postpartale Anwesenheit eines Pädiaters [16] - auch Dienstleistungen verschiedenster Art. Hier zeigt sich, dass Sicherheit und Sorge um die Gesundheit von Mutter und Kind selbstverständlich ein wichtiger Faktor sind, gleichzeitig aber natürlich auch noch andere (vermeintlich profanere) Gegebenheiten der Umgebung in deren Beurteilung einfließen. 


\section{Ausstattung und Service}

Stahl etwa fand heraus, dass die Erfüllung nicht-medizinischer Dienstleistungen ein häufiger Anlass zur Unzufriedenheit der befragten Frauen war, so wie beispielsweise bei der mangelhaften Vorbereitung auf die Klinikentlassung und beim Essen [12]. Auch Ausstattung und räumliche Voraussetzungen, unter anderem Umgebung und Atmosphäre während der Geburt [7] und die Zimmeratmosphäre [12] erscheinen als wichtiger Bezugspunkt für eine positive Beurteilung des Geburtsortes. Hier wurde innerhalb Deutschlands in den vergangenen Jahren den Wünschen der Frauen bereits Rechnung getragen, indem aufwändige Kreißsaal-Sanierungen an vielen Stellen ausgeführt wurden. Trotzdem erstreckt sich der Begriff Atmosphäre über räumliche Gegebenheiten hinaus - der Duden erwähnt etwa neben der tatsächlichen Umgebung unter anderem noch Ausstrahlung und Stimmung als Bedeutung [13].

\section{Information und Aufklärung}

Zentral erscheinen außerdem Information und Aufklärung der Patientin/Klientin: So beklagen die Teilnehmerinnen der Untersuchung von Sandin-Bojö et al. mangelhafte Information über den Geburtsverlauf und Untersuchungsergebnisse [6], während bei Henderson mangelnde Information bereits präpartal im Fokus steht [8] und auch Groß die Zufriedenheit mit den erfahrenen Dienstleistungen, im Sinne angewendeter Maßnahmen, zu den wichtigsten Aspekten der Geburtserfahrung zählt [11]. Hier ist auch der Rückgriff auf den Begriff des Empowerments durchaus sinnvoll: Durch verschiedene Methoden (unter anderem die Aufklärung und Miteinbeziehung von Klientinnen und Patientinnen) soll deren Selbstbestimmtheit und Mündigkeit gefördert werden [18]. Diese Mündigkeit wird heute nicht nur im Bereich der Geburtshilfe gefordert. Aber gerade hier, wo es um junge Menschen in der Mitte ihres Lebens geht, die zudem noch lernen müssen, für einen weiteren Menschen die Verantwortung zu tragen, ist diese Selbstbestimmtheit von enormer Wichtigkeit.

\section{Beratung und Unterstützung}

Einen besonderen Stellenwert in der untersuchten deutschen Literatur haben für viele Frauen außerdem die Stillberatung und -unterstützung sowie die Beratung im Wochenbett: Bezogen auf hilfreiche Aspekte der Hebammenbetreuung erwähnten Frauen in der Untersuchung von Ahrendt et al., die „Beratungskompetenz (...) als besonders bedeutsame Fähigkeit der Hebamme“, sowie die Unterstützung bei der Säuglingspflege [15]. Als unzureichend allerdings wurde in der Studie von Stahl an zweithäufigster Stelle die Stillunterstützung empfunden [12], was besonders besorgniserregend ist, wenn man betrachtet, dass das Bedürfnis nach Hilfe in diesem Bereich von Frauen bereits präpartal als besonders wichtig angegeben wird [16]. Da immer wieder nach Gründen für eine mangelnde Bereitschaft zum (Weiter-)Stillen gesucht wird, haben viele Kliniken durch Unterstützung nach IBCLC zertifizierte Beraterinnen gesucht und bisweilen auch den mühsamen
Weg zum stillfreundlichen Krankenhaus/baby-friendly-hospital auf sich genommen. Hier scheint der Ansatz, bereits ab der Geburt umfassende Stillunterstützung zu geben, dem Wunsch der Frauen entgegenzukommen. Gleichzeitig müssen auch die Ansätze, die eine Stillvorbereitung in der Schwangerschaft bieten, deutlich gefördert und publik gemacht werden. Denn auch an dieser Stelle gilt der Grundsatz des Empowerments, wobei Frauen und Paare durch umfassende Vorbereitung unabhängiger von den individuell sehr unterschiedlichen Rahmenbedingungen werden.

\section{Einladende Atmosphäre}

Zusammenfassend also lässt sich zum Bereich der Rahmenbedingungen sagen, dass Frauen sich einen Ort wünschen, an dem ihnen bei Bedarf medizinische Hilfeleistungen aller Art angeboten werden. Um der Physiologie der Geburt gerecht zu werden, muss man aber davon ausgehen, dass diese Angebote nicht im Vordergrund stehen sollen. Vielmehr sollen Räumlichkeiten und medizinisches Personal eine Atmosphäre schaffen, die dazu einlädt, sich auf die Geburt einzulassen, zur Familie zu werden und dabei die individuell nötige Unterstützung zu erhalten. Zugleich ist aber auch die individuell nötige Ruhe und Privatsphäre zu respektieren.

\section{Emotionale Erfahrungen}

Selbstverständlich ist eine Geburt begleitet von intensiven emotionalen Erfahrungen - diese reichen von überschäumendem Glück bis hin zum anderen Extrem der Traumatisierung der Beteiligten. Bei aller Vielseitigkeit der emotionalen Erlebnisse ist davon auszugehen, dass diese im Rückblick auf die Geburt immer einen sehr hohen Stellenwert einnehmen werden.

\section{Einbezogenheit und Mitsprache}

Frauen wissen dies und stellen deshalb bereits präpartal Forderungen an ihren Geburtsort: An Henderson et al. wurde der Wunsch nach Mitsprachemöglichkeiten bereits in der Schwangerschaft herangetragen [8]. Dieser äußerte sich auch postpartal im Bedürfnis nach Einbezogenheit in Entscheidungen [12][6]. Genannte Mitsprachemöglichkeiten überschneiden sich mit dem Term des Empowerments, welcher für die Rahmenbedingungen verwendet wurde. Damit wird aber auch klar, dass keine absolute Abgrenzung der Themen möglich und auch nicht gewünscht ist - diese wiederholen sich und wirken aufeinander ein. So kann die geforderte Teilnahme und Übernahme von Entscheidungen zu einem größeren Sicherheitsgefühl führen.

\section{Persönliche Kontrolle}

Dieses Gefühl äußerte sich z.B. auch in der Untersuchung von Cipolletta und Balasso [7], in der Frauen als besonders positive Erfahrung ein erlebtes Vertrauen in medizinische Interventionen und in diesem Zusammenhang ein gewisses Maß an wahrgenommener persönlicher Kontrolle betonten. Zudem stellten Guittier et al. fest, dass die 
Kontrolle zwar bei verschiedensten Geburtsmodi von den Frauen als positiv erlebt wurde, ausgenommen waren aber die Frauen mit Notkaiserschnitt, denen das Entgleiten der Kontrolle in der Notfallsituation als besonders negativ in Erinnerung geblieben war [9].

\section{Sicherheitsbedürfnis}

Empfindungen bezüglich Sicherheit und Vertrauen mit großem Einfluss auf Geburtserleben beschrieben Gärtner et al. [10]. Damit bestätigten sie Ahrendt et al., in deren Untersuchung die Vermittlung von Sicherheit durch die Hebamme bereits zentrales Anliegen der Frauen war, ebenso Stahl, in deren Studie das Eingehen auf Ängste und Sorgen thematisiert wurde [12]. Ebenso drückten die befragten Frauen gegenüber Tuschy et al. ein starkes Sicherheitsbedürfnis aus [16]. Als stützende Faktoren für empfundene Sicherheit könnte unter anderem die Bewahrung von Natürlichkeit im Rahmen der Klinikgeburt [14] sowie das Festhalten an einem interventionsarmen Vorgehen führen, wie sich in den Niederlanden zeigte Frauen, die ihr Kind im primären (interventionsarmen) System gebaren, zeigten höhere Zufriedenheit [5].

\section{Vertraute Begleitperson}

Tuschy et al. fanden außerdem heraus, dass Frauen schon in der Schwangerschaft beim Gedanken an die Geburt die Anwesenheit einer vertrauten Begleitperson besonders wichtig war [16]. Auch der Überblick von Groß stellte Beziehungsfaktoren in den Mittelpunkt [11]. Diese Forderung nach vertrauten Begleitpersonen ist heute zwar immer noch wichtig, aber glücklicherweise muss sie nur noch selten mit Nachdruck durchgesetzt werden. Vielmehr ist die Anwesenheit der Begleitperson eine Selbstverständlichkeit geworden. Hier allerdings gilt es, darauf zu achten, dass auch Frauen, die nicht vom Vater des Kindes begleitet werden (können), auf die Möglichkeit hingewiesen und dabei unterstützt werden, Vertrauenspersonen mitzunehmen.

\section{Erfüllte Erwartungen}

Übergeordnet war außerdem die ganz prinzipielle Forderung nach der Erfüllung von Erwartungen bei Christiaens und Bracke der beste Prädiktor für Zufriedenheit mit der Geburt [4]. Zu diesen Erwartungen darf sicher die postpartale Erfülttheit im Rahmen der individuellen Geburtserfahrung gezählt werden [11]. Auch ein sinnesübergreifendes Kennenlernen des Kindes war bei vaginaler Geburt einer der Faktoren, der die Zufriedenheit besonders positiv beeinflusste - ebenso zeigte sich aber auch, dass die Abwesenheit von mütterlichen Gefühlen während der ersten Tage postpartal zu großer Verunsicherung und Sorge führte [9].

\section{Herausforderung für das medizinische Personal}

So ist zu sehen, dass die Bandbreite der emotionalen Erfahrungen, die das Geburtserleben beeinflussen, groß ist. Gleichzeitig sind auch die Möglichkeiten, diese
Erfahrungen in positiver Weise zu beeinflussen, für das medizinische Personal groß: Sicherheit vermitteln, Erlebnisse und Entscheidungen verstehbar machen und Raum lassen für die individuellen emotionalen Geschehnisse im Rahmen der Geburt.

\section{Erlebte Betreuung}

Ein weiterer sehr zentraler Faktor in den bisher vorgestellten Erhebungen ist die erfahrene professionelle Betreuung im Rahmen der Geburt. Diese ist sehr weit gefächert und reicht von ärztlicher Begleitung über die Hebammenbetreuung bis hin zur Unterstützung durch Kinderschwestern, Stillberaterinnen und viele mehr.

\section{Wahrgenommene Unterstützung wichtig}

So stellten unter anderem Christiaens \& Bracke fest, dass wahrgenommene Unterstützung durch die Hebamme zu wahrgenommener persönlicher Kontrolle führte und in Zusammenhang mit einem hohen Maß an Selbstwirksamkeit stand [4]. Auch hier wiederholen sich die Begrifflichkeiten und es zeigen sich zudem vermittelnde Effekte und betonen die Verantwortlichkeit von Betreuungspersonen unter der Geburt. Gestützt werden diese Schlüsse zudem durch die Studie von Wiegers, die bekannte Betreuungspersonen als hilfreich für hohe Zufriedenheit identifizierte [5]. Auch Guittier et al. identifizierten die Aktionen und Reaktionen des geburtshilflichen Personals als besonders einflussreich [9] und Stahl sowie Gärtner et al. bezeichneten das Verhältnis des Pflegepersonals zur Frau sogar als zentral [12][10].

\section{Wertschätzung von Wünschen}

Ebenfalls vermittelnde Faktoren identifizierte Groß, indem sie die Teilhabe und Kontrolle über das Geschehen als wichtige Punkte für gebärende Frauen bezeichnete und daraus Aufgaben der Hebammen während der Geburt (im Sinne von Aufklärung und Unterstützung der Selbstbestimmung durch Einbeziehung in Entscheidungen) ableitete [11]. Diese Wertschätzung von individuellen Wünschen betonten Frauen auch vorausschauend im Hinblick auf die bevorstehende Geburt als besonders wichtig [14]. Durch die kontinuierliche Betreuung durch eine Hebamme peri- und postpartal erhofften sich die Frauen eine Wahrung ihrer Bedürfnisse [16].

\section{Individuelle Betreuung statt Routinen}

Während genannte Untersuchungen die positiven Effekte durch professionelle Betreuung unterstrichen, zeigten sich unter anderem bei Sandin-Bojö deutliche Kritikpunkte [6]: Frauen wünschten mehr Wertschätzung durch das Personal und mehr individuelle Pflege statt Routinen. Außerdem war das allgemeine Bedürfnis nach Unterstützung ein wichtiges Thema [7]. Kritisch wurde auch bei Stahl vor allem das mangelhafte Eingehen auf Ängste und Sorgen gesehen, ebenso die insgesamt häufig fehlende Einbindung in Entscheidungen. 
Diese wurde in allen Gruppen des Betreuungspersonals bemängelt, z.B. durch unzureichende Information und Stillberatung, die sogar als schwerer Mangel empfunden wurde [12]. Auch bei Guittier et al. kristallisierte sich die Bedeutsamkeit der Aktionen und Reaktionen des geburtshilflichen Personals heraus, welche sowohl positiven als auch negativen Einfluss auf die Verarbeitung der Situation durch die Schwangere hatte [9].

\section{Bedingungen für den optimalen}

\section{Geburtsort}

Übereinstimmend zeigte sich, dass die Rahmenbedingungen für eine positive oder auch verbesserungswürdige Erfahrung eine gewisse Rolle spielen. Dazu gehören der Literatur entnommene Themen wie Dienstleistung und räumliche Ausstattung [11][16], Aufklärung und Beratung [6][8] sowie Selbstbestimmtheit im Sinne von Empowerment [11][12].

Da Empowerment auch im Zuge der Dienstleistungen Aufklärung und Beratung erfolgt, und die genannten Begriffe als Gegebenheiten im Rahmen des Settings beschrieben werden können, erscheinen die Begriffe hier zusammengefasst unter dem Oberbegriff des Service-Komplexes.

Viele Nennungen gab es in den Untersuchungen zu den Begrifflichkeiten der wahrgenommenen persönlichen Kontrolle, bzw. reduzierten Interventionen und Sicherheit [4], zu Geburtserlebnis und Erfülltheit [4][9][11] [14] und vereinzelt auch zu Begleitpersonen [16]. All diese Nennungen werden hier subsummiert unter dem Begriff der emotionalen Erfahrungen, welche Frauen während einer Geburt machen.

Ebenso thematisiert wurden immer wieder das Betreuungspersonal und dessen Umgang mit der Frau. Hier waren Nennungen zur Wertschätzung [6], das Ausmaß und die Qualität von Betreuung im Sinne von Feedback für die Frau mit resultierenden positiven Ergebnissen [5][7][9][10][12][16] sowie empfundenes Selbstvertrauen [15] zu finden. Um die genannten Begrifflichkeiten zusammenzufassen, wurde der Begriff der erlebten Betreuung gewählt.

\section{Fazit}

Zusammenfassend lässt sich sagen, dass der Geburtsort nicht ausschließlich als räumlicher Ort, sondern zudem als Hort der Betreuung sowie als Platz, an dem emotionale Erfahrungen möglich gemacht bzw. aufgefangen werden sollen, zu betrachten ist. Frauen sollen in die Entscheidungen nicht nur mit einbezogen werden, sondern ihre aktuellen Wünsche und Bedürfnisse müssen das Handeln leiten - und zwar neben der 
>Tab. 1 Internationale Untersuchungen.

\begin{tabular}{|c|c|c|c|c|c|}
\hline Autoren & Studie & Forschungsfrage & Rahmenbedingungen & $\begin{array}{l}\text { Emotionale } \\
\text { Erfahrungen }\end{array}$ & Erlebte Betreuung \\
\hline $\begin{array}{l}\text { Christiaens \& } \\
\text { Bracke, } 2007\end{array}$ & $\begin{array}{l}\text { Länderübergreifender Ver- } \\
\text { gleich (Belgien, } \\
\text { Niederlande) } \\
\text { Quantitatives Design } \\
605 \text { TN } \\
\text { Längsschnitt- } \\
\text { Untersuchung }\end{array}$ & $\begin{array}{l}\text { Zufriedenheit der Frauen } \\
\text { in Abhängigkeit vom } \\
\text { System }\end{array}$ & & $\begin{array}{l}\text { Erfüllung von Erwar- } \\
\text { tungen zentral } \\
\text { gefordert }\end{array}$ & $\begin{array}{l}\text { Wahrgenommene Unter- } \\
\text { stützung durch die Heb- } \\
\text { amme führt zu } \\
\text { wahrgenommener per- } \\
\text { sönlicher Kontrolle und } \\
\text { steht in Zusammenhang } \\
\text { mit einem hohen Maß an } \\
\text { Selbstwirksamkeit }\end{array}$ \\
\hline Wiegers, 2009 & $\begin{array}{l}\text { Längsschnittliches Design } \\
600 \text { TN } \\
\text { Niederlande }\end{array}$ & $\begin{array}{l}\text { Evaluation des nieder- } \\
\text { ländischen } \\
\text { Betreuungssystems }\end{array}$ & & $\begin{array}{l}\text { Interventionsarmes } \\
\text { primäres System führt } \\
\text { zu höherer } \\
\text { Zufriedenheit }\end{array}$ & $\begin{array}{l}\text { Bekannte Betreuungsper- } \\
\text { sonen als hilfreich für } \\
\text { hohe Zufriedenheit }\end{array}$ \\
\hline $\begin{array}{l}\text { Sandin-Bojö } \\
\text { et al., } 2011\end{array}$ & $\begin{array}{l}\text { Quantitative postpartale } \\
\text { (14 Tage) } \\
\text { Querschnittbefragung } \\
700 \text { TN } \\
\text { Schweden }\end{array}$ & $\begin{array}{l}\text { Unterschiede zwischen } \\
\text { erlebter Betreuung und } \\
\text { deren persönlicher } \\
\text { Relevanz }\end{array}$ & $\begin{array}{l}\text { Informationen über den } \\
\text { Geburtsverlauf und } \\
\text { Untersuchungsergeb- } \\
\text { nisse } \\
\text { verbesserungsbedürftig }\end{array}$ & $\begin{array}{l}\text { Mehr Beteiligung an } \\
\text { Entscheidungen } \\
\text { gefordert }\end{array}$ & $\begin{array}{l}\text { Mehr Wertschätzung } \\
\text { angebracht durch Perso- } \\
\text { nal } \\
\text { Mehr individuelle Pflege } \\
\text { statt Routinen gewünscht }\end{array}$ \\
\hline $\begin{array}{l}\text { Cipolletta \& } \\
\text { Balasso, } 2011\end{array}$ & $\begin{array}{l}\text { Qualitatives Design } \\
10 \text { TN } \\
1-3 \text { Tage postpartal } \\
\text { Italien }\end{array}$ & $\begin{array}{l}\text { Erfahrungen erstgebä- } \\
\text { render Frauen bei der } \\
\text { Geburt }\end{array}$ & $\begin{array}{l}\text { Umgebung und Atmo- } \\
\text { sphäre während der } \\
\text { Geburt }\end{array}$ & $\begin{array}{l}\text { Wahrgenommene } \\
\text { Kontrolle } \\
\text { Vertrauen in die } \\
\text { medizinischen } \\
\text { Interventionen }\end{array}$ & $\begin{array}{l}\text { Bedürfnis nach } \\
\text { Unterstützung }\end{array}$ \\
\hline $\begin{array}{l}\text { Henderson, } \\
2013\end{array}$ & $\begin{array}{l}\text { Quantitatives epidemiologi- } \\
\text { sches Design } \\
24000 \text { TN } \\
\text { Querschnitt } \\
\text { England }\end{array}$ & $\begin{array}{l}\text { Relevante Einflussfakto- } \\
\text { ren auf die erlebte peri- } \\
\text { partale Betreuung bei } \\
\text { Migrantinnen }\end{array}$ & $\begin{array}{l}\text { Mangelnde Informatio- } \\
\text { nen präpartal }\end{array}$ & $\begin{array}{l}\text { Mitsprachrecht } \\
\text { gewünscht präpartal }\end{array}$ & \\
\hline $\begin{array}{l}\text { Guittier et al., } \\
2014\end{array}$ & $\begin{array}{l}\text { Qualitatives Design } \\
24 \text { TN } \\
\text { 4-6 Wochen postpartal } \\
\text { Schweiz }\end{array}$ & $\begin{array}{l}\text { Erfahrungen erstgebä- } \\
\text { render Frauen in Abhän- } \\
\text { gigkeit vom } \\
\text { Geburtsmodus }\end{array}$ & & $\begin{array}{l}\text { Entgleiten der Kon- } \\
\text { trolle (Notfall) } \\
\text { Sinnesübergreifen- } \\
\text { des kennenlernen } \\
\text { des Kindes (vaginale } \\
\text { Geburt) }\end{array}$ & $\begin{array}{l}\text { Aktionen und Reaktionen } \\
\text { des geburtshilflichen } \\
\text { Personals mit großem } \\
\text { Einfluss }\end{array}$ \\
\hline $\begin{array}{l}\text { Gärtner et al., } \\
2014\end{array}$ & $\begin{array}{l}\text { Mixed-Methods } \\
\text { Online Fokusgruppen- } \\
\text { diskussion } \\
\text { Expertinneninterviews } \\
\text { Fragebogenentwicklung } \\
\text { Niederlande }\end{array}$ & $\begin{array}{l}\text { Identifikation relevanter } \\
\text { Dimensionen des } \\
\text { Geburtserlebens }\end{array}$ & & $\begin{array}{l}\text { Empfindungen bezüg- } \\
\text { lich Sicherheit und } \\
\text { Vertrauen mit großem } \\
\text { Einfluss auf } \\
\text { Geburtserleben }\end{array}$ & $\begin{array}{l}\text { Geburtshilfliches Personal } \\
\text { zentral für Geburtserleben }\end{array}$ \\
\hline
\end{tabular}

optimalen Überwachung und medizinischen Betreuung. Dann ist eine gute Geburt möglich und sogar wahrscheinlich.

\section{Literatur}

[1] iresearch4birth: Babies Born Better Survey. http://www.iresearch4birth.eu/iResearch4Birth/en/bbb1.wp (last accessed on 11 March 2016).

[2] Luegmair, Karolina; Zenzmaier, Christoph, Oblasser, Claudia; König-Bachmann, Martina: Women's satisfaction with care at the birthplace in Austria: Evaluation of the Babies Born Better Survey national dataset In: Midwifery 59 (2018) 130-140.
[3] Makowsky Katja, Schücking Beate (eds.): Was sagen die Mütter: Qualitative und quantitative Forschung rund um Schwangerschaft, Geburt und Wochenbett. 1st ed. Weinheim: Beltz Juventa, 2013.

[4] Christiaens Wendy, Bracke Piet: Assessment of social psychological determinants of satisfaction with childbirth in a cross-national perspective In: BMC pregnancy and childbirth, 2007/7, 26.

[5] Wiegers Therese: The quality of maternity care services as experienced by women in the Netherlands In: BMC pregnancy and childbirth, 2009/9, 18.

[6] Sandin-Bojö Ann-Kristin, Kvist Linda, Berg Marie, Larsson Bodil: What is, could be better: Swedish women's perceptions of their intrapartal care during planned vaginal birth In: International journal of health care quality assurance, 2011/ 24, 81-95. 
-Tab. 2 Deutschsprachige Untersuchungen.

\begin{tabular}{|c|c|c|c|c|c|}
\hline Autoren & Studie & Forschungsfrage & $\begin{array}{l}\text { Rahmenbedingun- } \\
\text { gen }\end{array}$ & $\begin{array}{l}\text { Emotionale } \\
\text { Erfahrungen }\end{array}$ & Erlebte Betreuung \\
\hline Groß, 2003 & Überblicksarbeit & $\begin{array}{l}\text { Identifikation der } \\
\text { Wünsche und } \\
\text { Bedürfnisse } \\
\text { Gebärender }\end{array}$ & $\begin{array}{l}\text { Zufriedenheit mit } \\
\text { medizinischen } \\
\text { Dienstleistungen }\end{array}$ & $\begin{array}{l}\text { Allgemeine } \\
\text { Geburtserfahrung } \\
\text { Postpartale } \\
\text { Erfülltheit } \\
\text { Beziehungsfakto- } \\
\text { ren }\end{array}$ & $\begin{array}{l}\text { Aufklärung und } \\
\text { Unterstützung von } \\
\text { Selbstbestimmung }\end{array}$ \\
\hline Stahl, 2009 & $\begin{array}{l}\text { Retrospektive } \\
\text { Befragung } \\
\text { Quantitatives Design } \\
3000 \text { TN } \\
\text { Deutschland }\end{array}$ & $\begin{array}{l}\text { Entwicklung und } \\
\text { Evaluation eines } \\
\text { Fragebogens zur } \\
\text { Zufriedenheit mit } \\
\text { der Geburtsklinik }\end{array}$ & $\begin{array}{l}\text { Informationen und } \\
\text { Stillberatung }\end{array}$ & $\begin{array}{l}\text { Eingehen auf } \\
\text { Ängste und } \\
\text { Sorgen } \\
\text { Einbindung in } \\
\text { Entscheidungen }\end{array}$ & $\begin{array}{l}\text { Verhältnis des Pflege- } \\
\text { personals zur Frau } \\
\text { zentral }\end{array}$ \\
\hline $\begin{array}{l}\text { Hainer \& } \\
\text { Kowalcek, } \\
2011\end{array}$ & $\begin{array}{l}\text { Prospektives } \\
\text { Querschnittsdesign } \\
\text { Quantitativ } \\
534 \text { TN } \\
\text { Deutschland }\end{array}$ & $\begin{array}{l}\text { Wünsche von } \\
\text { Schwangeren } \\
\text { bezüglich des } \\
\text { Entbindungsmodus }\end{array}$ & & $\begin{array}{l}\text { Bewahrung von } \\
\text { Natürlichkeit im } \\
\text { Rahmen der } \\
\text { Klinikgeburt }\end{array}$ & $\begin{array}{l}\text { Wertschätzung von } \\
\text { individuellen } \\
\text { Wünschen }\end{array}$ \\
\hline $\begin{array}{l}\text { Ahrendt et al. } \\
2012\end{array}$ & $\begin{array}{l}\text { Quantitatives } \\
\text { Querschnittsdesign } \\
80 \text { TN } \\
\text { Deutschland }\end{array}$ & $\begin{array}{l}\text { Erwartungen und } \\
\text { Wünsche an die } \\
\text { Hebamme }\end{array}$ & Beratung & $\begin{array}{l}\text { Vermittlung von } \\
\text { Sicherheit }\end{array}$ & $\begin{array}{l}\text { Stützung des } \\
\text { Selbstvertrauens }\end{array}$ \\
\hline $\begin{array}{l}\text { Tuschy et al. } \\
2012\end{array}$ & $\begin{array}{l}\text { Prospektives quantita- } \\
\text { tives Design ab der } \\
\text { 20. SSW } \\
500 \text { TN } \\
\text { Deutschland }\end{array}$ & $\begin{array}{l}\text { Erwartungen und } \\
\text { Wünsche an die } \\
\text { ideale Geburtsklinik }\end{array}$ & $\begin{array}{l}\text { Stillberatung } \\
\text { Postpartale Anwe- } \\
\text { senheit eines } \\
\text { Pädiaters }\end{array}$ & $\begin{array}{l}\text { Sicherheitsbedürf- } \\
\text { nis } \\
\text { Anwesenheit einer } \\
\text { Begleitperson }\end{array}$ & $\begin{array}{l}\text { Kontinuierliche } \\
\text { Betreuung durch eine } \\
\text { Hebamme Peri-und } \\
\text { postpartal }\end{array}$ \\
\hline
\end{tabular}


[7] Cipolletta Sabrina, Balasso Silvia: When everything seems right: the first birth experience of women in an Italian hospital In: Journal of Reproductive and Infant Psychology, 2011/ 29, 374-381.

[8] Henderson Jane: Experiencing maternity care: the care received and perceptions of women from different ethnic groups In: BMC pregnancy and childbirth , 2013/13, e1e14.

[9] Guittier Marie-Julia, Cedraschi Christine, Jamei Nasir, Boulvain Michel, Guillemin Franics: Impact of mode of delivery on the birth experience in first-time mothers: a qualitative study In: BMC pregnancy and childbirth, 2014/14, 254-262.

[10] Gärtner Fania, Freeman Liv, Rijnders Marlies, et al.: A comprehensive representation of the birth-experience: identification and prioritization of birth-specific domains based on a mixed-methods design In: BMC pregnancy and childbirth, 2014/14, 147-157.

[11] Groß Mechthild: Die Geburtserfahrung-eine Übersichtsarbeit In: Geburtshilfe und Frauenheilkunde, 2003/63, 321-325.

[12] Stahl Katja: Wie zufrieden sind Frauen mit ihrer Geburtsklinik? In: Zeitschrift für Geburtshilfe und Neonatologie , 2009/213, 11-17.

[13] Duden: Rechtschreibung. www.duden.de (last accessed on 19 April 2016).

[14] Hainer F., Kowalcek Ingrid: Wünsche von Schwangeren an den Geburtsmodus In: Zeitschrift für Geburtshilfe und Neonatologie, 2011/215, 35-40.

[15] Ahrendt Cordula, Krauss-Lembcke Sabine, Luther Birte, Reutter Renate: Erwartungen und Wünsche an die Hebamme - Ergebnisse einer Umfrage unter jungen Müttern In: Die Hebamme, 2012, 226-232.
[16] Tuschy Benjamin, Berlit S., Hägele F., et al.: Welche Erwartungen stellen Schwangere an eine ideale Entbindungsklinik? In: Zeitschrift für Geburtshilfe und Neonatologie, 2012/216, 246-252.

[17] Knape Nina, Mayer Herbert, Schnepp Wilfried, Sayn-Wittgenstein Friederike: The association between attendance of midwives and workload of midwives with the mode of birth: secondary analyses in the German healthcare system In: BMC pregnancy and childbirth, 2014/14, 300-312.

[18] Rappaport Julian: Terms of empowerment/exemplars of prevention: Toward a theory for community psychology In: American Journal of Community Psychology, 1987/15, 121-148.

\section{Autorinnen/Autoren}

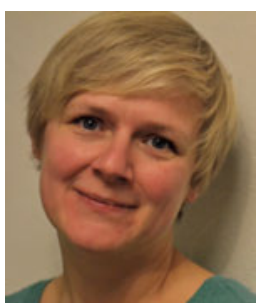

Karolina Luegmair ist Hebamme, B.Sc. Psychologie, M.Sc. Midwifery und Lehrerin an der Hebammenschule des BBZ Ingolstadt

\section{Korrespondenzadresse}

\section{Karolina Luegmair}

85777 Fahrenzhausen

E-Mail: karolina.luegmair@gmx.de 